remarkable, as death usually occurs within forty-eight hours; and it may be a question whether the external injury above referred to, or the arnica, could have had anything to do with the formation of the ulcers, inasmuch as there were some gastric symptoms before these suspected causes occurred.

\section{ENUCLEATION OF THE EYEBALL.}

By P. Pineo, M.D., of Hyannis.

Mrs. S., aged 64, was seized with inflammation of the right eye, in March, 1868. In July, 1868, I was first called to see her, at which time she was suffering much pain and discomfort from a staphylomatous condition of the cornea and inflammation of nearly all the tissues of the eyeball. The patient had been attended by an " Eclectic Doctor" until after the disorganization of the eye and loss of sight, and I failed to get any intelligent description of the character of the inflammation in its earlier stages, or of the progress of the disease. She probably had had irido-keratitis, with sloughing of the cornea, producing the condition present.

For the conjunctivitis, a collyrium of biborate of soda was prescribed ; for the pain, a solution of sulphate of atropia. She became more comfortable, yet suffering more or less, until January, 1869, when an increase of pain and inflammation occurred.

On again being called, I found distention of the eyeball, inflammation of its tissues, with severe pain, not only in the disorganized eye, but in the orbit of both eyes, with photophobia of the sound eye, which symptom had always been present more or less. Evidences of sympathetic trouble in the sound eye were unmistakeable, promising to involve the loss of sight.

February 10th, I punctured the corneal cicatrix, giving exit to a purulent discharge, which gave some relief from the distention, although the pain and distress continued. At length I advised and strongly urged, what at first had been suggested, removal of the eyeball.

On the 20th inst., enucleation of the eyeball was done, with the assistance of Dr. George, of Centreville. The conjunctiva, which was greatly thickened, was first divided close to the cornea; the tendon of the internal rectus was then divided, and successively the other recti and the oblique muscles, and with a pair of small curved seissors the optic nerve was cut close to the globe, when the eye rolled from its socket, leaving the capsulse of Tenon unharmed. Scarcely a drop of blood was lost, except that which came from the engorged conjunctiva.

The patient slept quietly, under the influence of ether, during the operation, and, when restored to consciousness, very little discomfort was felt in the orbit. She rested well the following night-better than for many weeks before.

On the 21 st inst. she was perfectly comfortable and happy, with no discomfort about the eye, and promising a speedy recovery.

The after-treatment consists in a single thickness of cotton cloth over the lids, kept wet with cold water.

March 12th.--The orbit and lids healthy, and ready for an artificial eye. The patient has had no pain since the operation. The remaining eye is quite tolerant of light, and gaining strength.

February 22, 1869.

[We are indebted to Dr. J. B. S. Jackson for the following additional particulars. -ED.]

This specimen was exhibited in a fresh state to the Society for Medical Improvement. Dr. Coolidge having examined it, he subsequently reported that the retina was thickened but soft to the feel, of an opaque white color, detached from the choroid coat except at the entrance of the optic nerve, and extending in a funnel-shaped form about as far forward as the ciliary process. The same form of disease occurs in the brain, and was first described by Virchow as glioma. In its nature there is nothing malignant. The choroid showed some signs of inflammation. The capsule of Tenon was inflamed and adherent to the sclerotic; this last being healthy.

In a note received by Dr. Jackson, March 27 th, Dr. Pineo states that on cutting open the eye, he found it "filled with a pus-like semi-fluid mass."

\section{PIN WORMS, AND THEIR HOMOEOPATHIC} TREATMENT.

By Charles E. Buckingham, M.D.

The New England Medical Gazette for February, 1869, contains an article on Entozoa, which is a light not to be carelessly placed under a bushel, if its merits be equal to the Editor's comments-" one of the most important discoveries of modern medicine."

Let us have the whole history, for it took two to discover it. Mr. E. C. Haserick, of 